\title{
Corea del Norte: ¿hacia la apertura y la reunificación con Corea del Sur?
}

DOI: $10.32870 /$ mycp.v7i22.227

José Jaime López Jiménez*

\section{I} ntroducción

El tradicional hermetismo que ha caracterizado al gobierno de Corea del Norte, el último país considerado como de régimen comunista puro en el mundo, ha impedido verificar si efectivamente Pyongyang se ha decidido finalmente a introducir reformas en su economía planificada o si algunas acciones emprendidas recientemente (como los aumentos en los salarios y en los precios) son sólo medidas desesperadas para controlar el mercado negro de alimentos que ha crecido rápidamente como consecuencia de las graves hambrunas que han asolado a este país en la última década.

El presente trabajo da cuenta de algunos de los acontecimientos más relevantes que han estado ocurriendo últimamente en territorio norcoreano y que parecen apuntar hacia un cambio en la conducción de la economía por parte del régimen comunista, y su probable apertura al exterior como lo hizo China, así como también de algunos sucesos relevantes que tienen que ver con un mayor acercamiento con Corea del Sur, los cuales pudieran interpretarse como los primeros pasos hacia la reunificación de los dos países.

\section{Los cambios en el terreno económico}

No obstante que ni el gobierno de Corea del Norte ni sus medios de comunicación oficiales han informado absolutamente nada acerca de ello, fuentes diplomáticas de Pyongyang han filtrado a algunos medios de información sobre pequeños cambios en los férreos controles del gobierno sobre el mercado, los cuales, debido a la rigidez y el inmovilismo que han ca-

* Profesor investigador del Departamento de Estudios del Pacífico, de la Universidad de Guadalajara. racterizado al régimen comunista, bien pudieran considerarse como significativos e interpretarse como las primeras señales del cambio. Aún así, es muy pronto para establecer que estemos presenciando el inicio de reformas económicas, es decir, del comienzo de la transformación de la última economía totalmente planificada que queda en el mundo hacia la economía de mercado, como lo hicieron en su momento China (en 1979, bajo el régimen de Den Xiao-Ping) y Rusia (en 1989, con Mijail Gorvachev). De ahí que francamente son más las interrogantes y dudas que los datos concretos disponibles sobre lo que está ocurriendo en realidad en la Corea del Norte del controvertido líder Kim Jong II.

Lo cierto es que, a partir de julio de 2002, súbitamente se registraron importantes aumentos en los salarios y en los precios de los alimentos y otros productos básicos en Corea del Norte (de hecho, los únicos a los que tienen acceso los norcoreanos, y no precisamente en forma permanente). En efecto, de acuerdo con información que se ha dado a conocer en algunos medios, los precios y los salarios se han multiplicado por diez y por veinte respectivamente, lo que en apariencia constituye un esfuerzo por estimular la circulación del dinero. De ser así, se estaría alentando la oferta y la demanda, que a su vez son los pilares de la economía de mercado, en sustitución de los rígidos controles estatales sobre estas variables.

No hay que olvidar que la economía de Corea del Norte está en su totalidad controlada por el Estado, y casi toda la población, a excepción de los altos funcionarios del régimen, sobrevive con una ración diaria de arroz que se vende en los centros de distribución oficiales. Con la desaparición del bloque socialista y las reformas económicas emprendidas 
por China, Pyongyang se quedó prácticamente sola al debilitarse su relación con sus antiguos socios políticos y comerciales, lo que, aunado a la incidencia de sequías periódicas, la llevaron a padecer constantes crisis alimentarias durante los años noventa.

Bajo estas circunstancias, para la gran mayoría de los norcoreanos comer o no ha dependido básicamente de la ayuda humanitaria que han estado recibiendo del exterior en los últimos años y, en menor medida, del modelo de agricultura doméstica de subsistencia que practican: los campesinos separan una parte de la producción para su consumo y otro tanto lo venden por su cuenta en el mercado negro. Cabe señalar que son pocos los campesinos que han logrado acumular cierto capital y que se han visto beneficiados por algún funcionario, los cuales se las han ingeniado para cultivar pequeños huertos urbanos y cuyos productos son vendidos en el mercado negro.

Lo anterior se ha traducido en una mayor oferta de alimentos para la población, aunque a precios relativamente más altos. La tolerancia del gobierno hacia este mercado paralelo en cierta forma se ha visto acompañada con la repentina introducción de aumentos en los precios de los productos básicos, con la intención de que el dinero que fluya en el mercado negro pueda ser gastado después en el mercado oficial. Con ello, se podría decir que para el régimen comunista es más rentable aceptar ese mercado que intentar combatirlo, puesto que en gran medida ha venido a mitigar la escasez de alimentos en el mercado interno.

Aún así, no se puede afirmar que Corea del Norte se esté encaminando ya hacia un esquema de precios libres o de liberalización del mercado de alimentos. Quienes están analizando con mayor detenimiento estos cambios, intentan comparar esta situación con el modelo de transformación económica aplicado en China, que en su fase inicial permitió vender los excedentes agrícolas de los campesinos en el mercado libre, lo que se tradujo en mejores condiciones de vida en el medio rural y sentó las bases para el posterior desarrollo de la economía de mercado.

Empero, es necesario destacar la coincidencia del ingreso del más antiguo y firme aliado de Corea del Norte, China, a la Organización Mundial del Comercio (omc) desde 2001, con la cada vez más clara resignación del régimen de Kim Jong II, a la realidad de que no puede prolongar por más tiempo el aislamiento total de su país del exterior. En este sentido, se ha observado que uno de los síntomas más evidentes de las difíciles condiciones de vida y de supervivencia que prevalecen en Corea del Norte, son las crecientes fugas de ciudadanos a través de la frontera con China y sus intentos desesperados por refugiarse en las delegaciones diplomáticas asiáticas u occidentales en Pekín.

\section{Los cambios en el terreno político}

También en el plano político se han observado algunas señales de cambio en la tradicional actitud beligerante de Pyongyang, principalmente con su vecina Corea del Sur y con Estados Unidos. A este respecto, como una muestra de que Kim Jong II estaba dispuesto a reconciliarse con occidente, manifestó su disposición a reunirse con el entonces presidente de Corea de Sur, Kim Dae-jung, en el año 2000, lo que 
fue calificado como un hecho histórico en la conflictiva relación entre las dos coreas y acrecentó el optimismo en torno a una distensión política y militar y un acercamiento entre ambas naciones.

Posteriormente, tras el lamentable incidente en que se vieron involucradas las marinas de guerra de ambos países, el gobierno surcoreano adoptó una actitud conciliadora con Pyongyang ante este suceso, en el que murieron cuatro marinos de ese país y un número aún desconocido de marinos norcoreanos, propiciando la reanudación de los contactos ministeriales.

En 2002, como otro gesto más de la flexibilización de la postura de Corea del Norte ante occidente, Pyongyang aceptó celebrar una reunión entre el jefe de la diplomacia norteamericana (el secretario de Estado, Colin Powell), con su homólogo norcoreano, Paek Nam-sun, en Brunei, en el marco de la reunión de los países de la Asociación de Naciones del Sureste de Asia (ASEAN, por sus siglas en inglés). Dicha reunión representó el primer contacto de alto nivel entre Kim Jong II y el gobierno estadounidense. Este hecho fue especialmente significativo después de que el presidente George W. Bush desde el inicio de su gestión incluyera a Corea del Norte, junto con Iraq e Irán, en un supuesto "Eje del Mal", países que según la Casa Blanca han estado patrocinando el terrorismo internacional.

No obstante, a tres años de los atentados del 11 de septiembre en Estados Unidos, la campaña norteamericana contra ese "Eje del Mal" se ha concentrado en mayor medida sobre Medio Oriente; con el derrocamiento del régimen Talibán en Afganistán, la invasión de su vecino Iraq para derrocar a Sadam Hussein y con las veladas amenazas sobre Irán en torno al supuesto programa nuclear secreto de este país. Hoy la principal preocupación del gobierno norteamericano es el programa nuclear secreto de Pyongyang, principalmente tras la aceptación a fines de 2003 de la existencia de éste por parte del régimen de Kim Jong II, y que desencadenó una crisis nuclear en la región, en especial con Corea del Sur y Japón.

Hasta ahora, parece ser que la amenaza militar nuclear norcoreana ha sido más que nada un arma esgrimida por el gobierno comunista para conseguir más ayuda del exterior. De hecho, Pyongyang ha aceptado entrar en negociaciones con el gobierno estadounidense, a instancias de la diplomacia china, para tratar de resolver la crisis nuclear a cambio de que la Casa Blanca cese lo que los norcoreanos consideran como actitudes hostiles contra su país y promueva nuevas medidas de ayuda de occidente para la economía norcoreana.

Desde mediados de 2003, como una muestra más del creciente acercamiento entre las dos coreas, sucedió un acontecimiento que hasta hace poco parecía impensable: los dos países por fin quedaron unidos gracias a una doble conexión ferroviaria. En la costa occidental se inauguró una línea ferroviaria que une directamente a las dos capitales, mientras que en la costa oriental se puso en marcha otra línea que enlazará el Puerto de Pusan (Corea del Sur), con la frontera de Corea del Norte y el extremo oriente de Rusia. Esto ha dado como resultado un mayor acercamiento entre las dos coreas que, entre otras cosas, se ha traducido en un aumento de las visitas de reunificación familiar y que, posiblemente, representa el derrumbe de la última frontera emanada de la guerra fría.

\section{¿Hacia la reunificación coreana?}

Es un hecho que la división del territorio coreano, al concluir la segunda guerra mundial, se decidió en el exterior en el contexto de la lucha ideológica entre las dos superpotencias, y se llevó a cabo sin el consentimiento de la población coreana. Poco antes de finalizar la guerra del Pacífico, Estados Unidos y la antigua Unión Soviética dividieron la Península de Corea por el paralelo 38 para conseguir la rendición de los japoneses, quienes habían convertido el territorio coreano en protectorado de Japón en 1905. Posteriormente, 
en junio de 1950, las fuerzas de Corea del Norte cruzaron la línea divisoria, iniciándose así la Guerra de Corea que duraría hasta 1953 y que causó innumerables bajas en los ejércitos de ambos países y en la población civil. De ahí que no se pueda afirmar que los coreanos sean los únicos responsables de la separación de su nación. Y si bien ellos son conscientes de que en un futuro próximo van a convertirse en los verdaderos protagonistas de la reunificación de sus estados, está claro que, cuando menos de parte del actual gobierno norcoreano, existe un abierto rechazo a cualquier tipo de injerencia del exterior en la probable unificación entre el Norte y el Sur.

Ahora bien, ¿mediante qué fórmula o mecanismo podría realizarse la reunificación coreana? Se ha debatido mucho sobre esta cuestión, puesto que resulta fundamental buscar un método unificador que sea positivo, viable, eficaz y, en la medida de lo posible, moderado en sus costos políticos y económicos. Los propios coreanos han formulado diversos proyectos. El primer dirigente norcoreano, Kim II Sung, propuso crear la llamada República Confederal de Koryo, en la cual el sistema político comunista del norte y el capitalista del sur no experimentarían mayores cambios. Bajo este esquema, el nuevo gobierno resultante de la unificación estaría compuesto por un número igual de delegados norcoreanos y surcoreanos.

Kim II Sung vislumbraba a la futura Corea como una nación, un Estado con dos sistemas y dos gobiernos; sin embargo, esta idea no prosperó debido a los fuertes obstáculos e implicaciones de carácter político. Frente a esta fórmula de reunificación, que puede ser o no compartida, los coreanos en general rechazan el uso de la fuerza militar como instrumento de unificación. Entre la población coreana aún está presente el doloroso capítulo de la cruenta guerra que padecieron y nadie desea repetir la tragedia porque una nueva confrontación seguramente llevaría a la destrucción total de la propia península. De hecho, hasta la misma Constitución de la República Popu- lar Democrática de Corea acepta la reunificación por medios pacíficos.

Los políticos surcoreanos, por su parte, también han aportado fórmulas para la reunificación, incluso algunos presidentes de Corea del Sur han contribuido con sus iniciativas en la procuración de mecanismos para alcanzar la unidad de los dos pueblos. Chun Doo Hwan, por ejemplo, formuló su política sobre la base del reconocimiento mutuo y la no intervención en asuntos internos. Posteriormente, Roh Tae Woo propuso una especie de Commonwealth o Comunidad Nacional Coreana. Kim Dae-jung rechazó en su momento una reunificación instantánea que implicara la simple absorción del Norte en beneficio del Sur.

No obstante, Pyongyang ha mostrado su recelo hacia las propuestas que prevén cierta equidad y reciprocidad en el proceso hacia la unidad, puesto que existe el fundado temor de que se desencadene una rápida absorción del Norte por parte del Sur, lo que a la postre propiciaría el derrumbamiento del régimen norcoreano de Kim Jong II. Empero, habría que considerar que bajo este escenario Seúl se vería obligado a emprender una reunificación acelerada que resultaría sumamente problemática, ya que supondría un alto costo para los contribuyentes surcoreanos, quienes tendrían que sufragar los costos del proceso. Además, quizá lo más preocupante, es que se crearían grandes flujos de migración descontrolada de norcoreanos empobrecidos hacia el Sur, en busca de mejores condiciones de vida y mayores oportunidades de empleo.

Cabe mencionar que, a pesar de los esfuerzos por reducir las tensiones políticas y de las innumerables acciones de colaboración económica de Corea del Sur hacia Pyongyang, los surcoreanos se sienten un tanto frustrados ante la revelación del programa nuclear secreto del régimen comunista, que en buena medida representa una amenaza para la estabilidad de toda la región asiática. De ahí que el actual presidente, Roh Moo Jun, haya modificado ligeramente su política hacia Corea del Norte, 
introduciendo nuevos elementos de reciprocidad, equivalencia y una mayor dosis de realismo, ante la amenaza que este hecho representa para la estabilidad y la paz entre las dos coreas y para la región en general.

Resulta difícil responder a la pregunta de cuál es el modelo más adecuado de reunificación de las dos coreas, ya que igualmente problemático ha resultado cualquier tipo de acuerdo entre Pyongyang y Seúl. Ahora bien, es evidente que no funcionarían otros modelos de reunificación nacional, por ejemplo, la reunificación alemana resultó muy costosa y tuvo que recibir ayuda complementaria de la Unión Europea; además, las dos alemanias estaban en condiciones económicas relativamente mejor que las dos coreas y no sufrieron una guerra civil como sucedió en suelo coreano. Por otra parte, antes de consumarse la unificación germana existió una amplia cooperación entre el lado oriental y el occidental, lo que facilitó aún más el acercamiento definitivo entre los alemanes. Por el contrario, la guerra coreana originó, junto al clima de guerra fría, un sentimiento de tensión y hostilidad a ambos lados de la frontera que ha resultado difícil de superar.

$\mathrm{Al}$ margen de la reunificación vietnamita, que se ejecutó mediante la fuerza militar, tampoco resulta recomendable seguir el ejemplo de la reunificación entre China y Hong Kong, ya que el planteamiento chino de "un país, dos sistemas" puede interesarle a Pyongyang, pero posiblemente no a Seúl. Cabe recordar que en Hong Kong se permite desarrollar la economía de libre mercado y un gobierno más o menos democrático, pero las fuerzas armadas y la política exterior están bajo el control de Pekín. De ahí que resulte difícil querer trasladar el modelo de unificación chino; además, Hong Kong es un pequeño territorio con un gran poder económico pero sin peso en la política internacional, cuya historia y desarrollo estuvieron vinculados durante muchos años al colonialismo británico. Por lo tanto, de seguir el ejemplo de China, ¿cuál de las dos coreas aceptaría renunciar a sus fuerzas armadas? ¿Cómo organizar dos siste- mas económicos antagónicos sin dificultades en un solo territorio? Incluso Taiwan ha rechazado seguir la experiencia unificadora entre China, Macao y Hong Kong.

Es necesario señalar que hay algunos puntos de consenso entre las dos coreas. En primer lugar, tanto Seúl como Pyongyang renuncian expresamente a la unificación por medio de la fuerza. En segundo lugar, previo a cualquier intento de unificación, coinciden en que deberán intensificarse los esfuerzos de cooperación económica para disminuir las diferencias en el grado de desarrollo económico entre ambos países. Como parte de esta cooperación, tendrán que intensificarse los contactos entre la población civil en los ámbitos social, cultural, educativo y deportivo, a efecto de que, en un futuro próximo, puedan presentar proyectos comunes ante los organismos internacionales y dar una mejor imagen de cohesión y espíritu de unidad. En tercer lugar, en el escenario de un único Estado y bajo una bandera común, se requerirán períodos de transición hacia modelos políticos de convivencia provisional, como puede ser una confederación o una débil federación.

Será necesario sensibilizar al contribuyente surcoreano, puesto que en él recaería buena parte del costo económico del proceso de unificación. Aunque también cabe la posibilidad de que algunos organismos internacionales (como el Banco Mundial, el Banco Asiático de Desarrollo o la Unión Europea) se solidaricen aportando recursos para que el costo de la reunificación nacional pueda repartirse de forma más amplia, pero aún se desconoce en qué proporción.

Lo que está claro es que no es fácil ni conveniente una rápida reunificación coreana, por lo que será necesario avanzar paso a paso. En todo caso se tendría que trabajar primero en alcanzar la reconciliación nacional, la paz y la seguridad nacional, lo que abriría el camino para incrementar la cooperación económica a través del comercio intercoreano, del aumento de las inversiones y de la ayuda humanitaria de Corea del Sur a Corea del Norte. Una 
vez alcanzadas estas metas, se podría seguir avanzando en la reunificación a través de diversos mecanismos de integración económica que han aplicado otros países. Uno de ellos podría ser la creación de una zona de libre comercio, la cual permitiría eliminar las barreras internas al comercio entre ambos países y abriría las puertas al establecimiento de una especie de mercado común que garantice la libre circulación de mercancías, capitales y mano de obra. En la medida en que se pueda consolidar $\mathrm{y}$ perfeccionar este $\mathrm{u}$ otro mecanismo de integración económica, que podría complementarse con una unión monetaria, será entonces más fácil aspirar a la fase final: la unión política.

Como quiera que sea, el camino hacia la reunificación coreana no se verá exento de problemas y obstáculos, puesto que resulta muy complicado desarrollar un marco económico en el cual coexistan el capitalismo y el comunismo. Lo anterior si se toma en cuenta que posiblemente ni Corea del Sur quiera renunciar al capitalismo, ni Corea del Norte desee abandonar totalmente el comunismo. Por lo pronto, lo que parece más probable es que Pyongyang continúe flexibilizando su control sobre la economía, tal como lo hizo en su momento China, y continúe con su cauteloso acercamiento con Seúl.

\section{Fuentes}

Asiared (2002 y 2003) Revista electrónica de actualidad de Asia, varios números, Barcelona, asiared@asiared.com.

Elúa Marta (1976) "República de Corea", Universidad de Deusto, Donosita.

Lucien Bianco (1976)Asia contemporánea, Siglo XXI Editores, México, DF.

Ojeda Alfonso (2004) “Análisis del futuro de la reunificación coreana", Conferencia pronunciada en el Congreso del Consejo para la Unificación Democrática y Pacífica de Corea, Madrid, 13 de marzo.

Romero Castilla Alfredo (2000) "La Península Coreana", en Asia Pacífico 2000, Centro de Estudios de Asia y África, El Colegio de México, junio.

Naewoe Press (1993) "North Korea: After Collapse of Socialist Camp”, Seúl, Corea. 